\title{
Dissipationless Vector Drag-Superfluid Spin Hall Effect
}

\author{
Andrzej Syrwid®, Emil Blomquist $\odot$, and Egor Babaev \\ Department of Physics, KTH Royal Institute of Technology, SE-106 91 Stockholm, Sweden
}

(Received 15 April 2021; revised 25 June 2021; accepted 6 August 2021; published 31 August 2021)

\begin{abstract}
Dissipationless flows in single-component superfluids have a significant degree of universality. In ${ }^{4} \mathrm{He}$, the dissipationless mass flow occurs with a superfluid velocity determined by the gradient of the superfluid phase. However, in interacting superfluid mixtures, principally new effects appear. In this Letter, we demonstrate a new kind of dissipationless phenomenon arising in mixtures of interacting bosons in optical lattices. We point out that for a particular class of optical lattices, bosons condense in a state where one of the components' superflow results in dissipationless mass flow of the other component, in a direction different from either of the components' superfluid velocities. The free-energy density of these systems contains a vector productlike interaction of superfluid velocities, producing the dissipationless noncollinear entrainment. The effect represents a superfluid counterpart of the Spin Hall effect.
\end{abstract}

DOI: 10.1103/PhysRevLett.127.100403

In 1975 Andreev and Bashkin demonstrated the principally new dissipationless drag-transport effect in an interacting superfluid mixture [1]. Namely, they showed that a nonzero superfluid velocity of one component induces a collinear dissipationless mass transfer of the other component. This entrainment effect is present in superfluids, superconductors-including those with unconventional pairing [2]—and dense nuclear matter [3,4]. It determines observed dynamics of pulsars [5-7] and can cause phase transitions to new types of superfluids [8-13].

As shown in [1], in the presence of intercomponent interactions, the free-energy density describing a binary superfluid mixture should necessarily include a scalar product of the superfluid velocities $\mathbf{v}_{a}$ and $\mathbf{v}_{b}$, i.e., $f=\rho_{a} v_{a}^{2} / 2+\rho_{b} v_{b}^{2} / 2+\rho_{a b} \mathbf{v}_{a} \cdot \mathbf{v}_{b}$. The resulting superflow $\mathbf{j}_{a}=\partial f / \partial \mathbf{v}_{a}=\rho_{a} \mathbf{v}_{a}+\rho_{a b} \mathbf{v}_{b}$ indicates that even if $\mathbf{v}_{a}=0$, there will still be a nonzero superflow of component $a$ with the superfluid velocity $\mathbf{v}_{b}$.

In the condensed matter context, the effect became of great interest with the advent of optical lattices, which allow for precise control of strongly correlated superfluids $[14,15]$. The strength of the Andreev-Bashkin drag is controlled by the optical lattice parameters in combination with on-site interactions [16]. It was shown that the effect, in relative terms, can be arbitrarily strong and that the Andreev-Bashkin drag coefficient $\rho_{a b}$ can also become negative. In the latter case, one deals with a counterflow, where the flow of one component generates a mass flow of

Published by the American Physical Society under the terms of the Creative Commons Attribution 4.0 International license. Further distribution of this work must maintain attribution to the author(s) and the published article's title, journal citation, and DOI. Funded by Bibsam. the other component in the opposite direction [8-11]. It was pointed out that the effect should lead to the formation of new superfluid states where only dissipationless coflow (paired superfluids) or only counterflow (supercounterfluids) can exist [8-13,16-18]. At the same time, even relatively weak drag substantially changes rotational responses $[19,20]$.

In this Letter, we demonstrate the existence of a new dissipationless phenomenon in superfluid mixtures, where the dissipationless superfluid entrainment is not collinear with superfluid velocities. Namely, we show that the superflow-superflow interaction can, in general, be described by a nontrivial tensor $\rho_{\alpha \beta}^{i j}$ which enters the bilinear free-energy density:

$$
f=\frac{1}{2} \sum_{\alpha \beta} \sum_{i j} \rho_{\alpha \beta}^{i j} v_{\alpha}^{i} v_{\beta}^{j} .
$$

Here Greek subscripts and Roman superscripts, respectively, label components and Cartesian directions of the superfluid velocity vector $v_{\alpha}^{i}$ and the superfluid stiffness tensor $\rho_{\alpha \beta}^{i j}=\rho_{\beta \alpha}^{j i}$. The latter describes both kinetic $(\alpha=\beta)$ and drag $(\alpha \neq \beta)$ phenomena. While physical properties of the system are encoded in the tensor $\rho_{\alpha \beta}^{i j}$, as we elaborate below, a direct interpretation of individual coefficients may be deceptive since they depend on the choice of the coordinate system.

In this Letter we consider a two-dimensional twocomponent system-i,j $j \in\{x, y\}$ and $\alpha, \beta \in\{a, b\}$-and study the drag-related elements $\rho_{a b}^{i j}$. In such a case, the quantities

$$
\rho_{\|}=\left(\rho_{a b}^{x x}+\rho_{a b}^{y y}\right) / 2, \quad \rho_{\perp}=\left(\rho_{a b}^{x y}-\rho_{a b}^{y x}\right) / 2,
$$




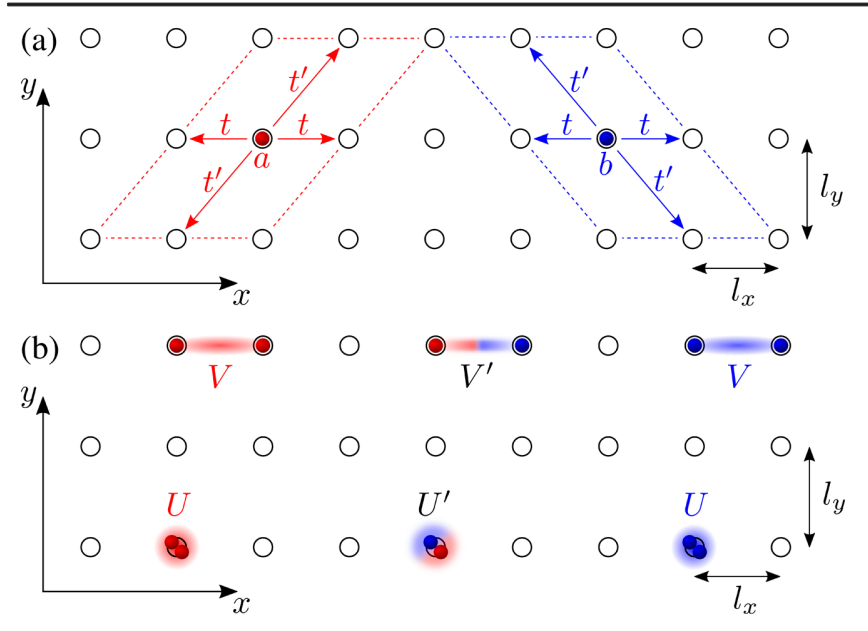

FIG. 1. Illustration of model parameters. (a) The allowed hopping directions. Both components can hop in the $x$ direction with the amplitude $t$. In addition, component $a$ (red) and component $b$ (blue) can hop along the diagonal and antidiagonal, respectively. The latter process is characterized by the amplitude $t^{\prime}$. Intra- and intercomponent interactions are schematically illustrated in (b).

are coordinate system independent [21]. All other pairwise combinations vary under rotation, and it turns out that it is always possible to find a Cartesian coordinate system in which $\rho_{a b}^{x y}+\rho_{a b}^{y x}=0$. If, in addition, the difference $\rho_{a b}^{x x}-$ $\rho_{a b}^{y y}$ is negligible - which in principle can be guaranteed in certain situations-one finds

$$
f=f_{0}+\rho_{\|} \mathbf{v}_{a} \cdot \mathbf{v}_{b}+\rho_{\perp}\left(v_{a}^{x} v_{b}^{y}-v_{a}^{y} v_{b}^{x}\right),
$$

where $f_{0}=\sum_{\alpha} \sum_{i j} \rho_{\alpha \alpha}^{i j} v_{\alpha}^{i} v_{\alpha}^{j} / 2$ represents the standard kinetic contribution to the free-energy density. The corresponding superflows read

$$
\begin{aligned}
& \mathbf{j}_{a}=\mathbf{j}_{0 a}+\rho_{\|} \mathbf{v}_{b}+\rho_{\perp}\left(v_{b}^{y} \mathbf{e}_{x}-v_{b}^{x} \mathbf{e}_{y}\right), \\
& \mathbf{j}_{b}=\mathbf{j}_{0 b}+\rho_{\|} \mathbf{v}_{a}-\rho_{\perp}\left(v_{a}^{y} \mathbf{e}_{x}-v_{a}^{x} \mathbf{e}_{y}\right),
\end{aligned}
$$

where $\mathbf{j}_{0 \alpha}=\partial f_{0} / \partial \mathbf{v}_{\alpha}$ and $\mathbf{e}_{i}$ denotes the unit vector in the $i$ th direction. The existence of the vector productlike contribution to $f$ given by $\rho_{\perp}$ constitutes a novel superfluid effect where a nonzero superflow of one component induces a perpendicular superflow response of the other component. This new phenomenon, which we coin vector drag, may be viewed as an intercomponent-interactiondriven counterpart of the Spin Hall effect [22] originating in the spin-orbit coupling, discussed particularly in hybrid structures involving superconductors [23,24].

Let us now provide both analytical and numerical evidence for the existence of a nonzero $\rho_{\perp}$, and thus the existence of the vector-drag phenomenon. Our starting point in deriving the effective model in Eq. (1) is a twocomponent Bose-Hubbard-type model on a rectangular lattice, given by the Hamiltonian

$$
\hat{H}=-\sum_{\alpha} \sum_{i j} t_{i j \alpha} \hat{b}_{i \alpha}^{\dagger} \hat{b}_{j \alpha}+\frac{1}{2} \sum_{\alpha \beta} \sum_{i j} U_{i j \alpha \beta} \hat{n}_{i \alpha} \hat{n}_{j \beta}
$$

Here $\hat{b}_{i \alpha}\left(\hat{b}_{i \alpha}^{\dagger}\right)$ denotes the bosonic annihilation (creation) operator of component $\alpha$ at site $i$, and $\hat{n}_{i \alpha}=\hat{b}_{i \alpha}^{\dagger} \hat{b}_{i \alpha}$ is the corresponding particle number operator. The mass of the $\alpha$ component boson is $m_{\alpha}$, and the rectangular lattice consists of $N \times N$ sites-with lattice vectors $\mathbf{a}_{x}=l_{x} \mathbf{e}_{x}, \mathbf{a}_{y}=l_{y} \mathbf{e}_{y}$ and lattice constants $l_{x}, l_{y}$-on which we impose periodic boundary conditions. For simplicity, we assume constant particle number densities $n_{\alpha}$ and restrict ourselves to on-site and nearest-neighbor interactions whilst allowing for nearest-neighbor and next-nearest-neighbor hopping. The problem is amenable to analytical treatment only in the weakly interacting regime. Here, like in the case of the AndreevBashkin effect [25-29], the drag effects are expected to be inherently small. We will first demonstrate the existence of vector drag in the weakly interacting regime analytically. Then we investigate the effect in the strongly correlated regime by employing large-scale quantum Monte-Carlo calculations.

The standard Andreev-Bashkin effect can be analytically calculated in macroscopic weakly interacting systems. That was previously done for square and triangular lattices and in a continuum [25-28]. We begin by employing a similar analytic approach to establish the new phenomenon: the vector drag. Consider a weakly interacting regime where at low enough temperatures both components are condensed into the zero-momentum mode. In such a case, the Hamiltonian [Eq. (5)] can be approximated and subsequently diagonalized in momentum space. The corresponding zero-temperature free-energy density [Eq. (1)] is then obtained as the ground state energy.

As discussed above, the vector drag should be the most transparent when $\rho_{a b}^{x y}=-\rho_{a b}^{y x}$, which is guaranteed for systems being invariant under a reflection in either of the two lattice vectors combined with an exchange of components $a \leftrightarrow b$ [21]. Our aim is to construct a microscopic model which exhibits $\rho_{\perp} \neq 0$ in addition to satisfying the above-mentioned symmetry. A simple choice of parameters obeying these conditions is $m_{a}=m_{b}=m$, $n_{a}=n_{b}=n$, and

$$
\begin{gathered}
t_{i j \alpha}= \begin{cases}t & \text { for } \mathbf{r}_{i}=\mathbf{r}_{j} \pm \mathbf{a}_{x} \\
t^{\prime} & \text { for } \mathbf{r}_{i}=\mathbf{r}_{j} \pm \mathbf{a}_{x} \pm\left(\delta_{a \alpha}-\delta_{b \alpha}\right) \mathbf{a}_{y}, \\
0 & \text { otherwise }\end{cases} \\
U_{i j \alpha \beta}= \begin{cases}U \delta_{\alpha \beta}+U^{\prime}\left(1-\delta_{\alpha \beta}\right) & \text { for } \mathbf{r}_{i}=\mathbf{r}_{j} \\
V \delta_{\alpha \beta}+V^{\prime}\left(1-\delta_{\alpha \beta}\right) & \text { for } \mathbf{r}_{i}=\mathbf{r}_{j} \pm \mathbf{a}_{x}, \\
0 & \text { otherwise }\end{cases}
\end{gathered}
$$

where $\mathbf{r}_{i}$ indicates the $i$ th lattice site position. The resulting model is illustrated in Fig. 1. While in general $\rho_{a b}^{x x} \neq \rho_{a b}^{y y}$, it turns out that when both $\rho_{a b}^{x x}$ and $\rho_{a b}^{y y}$ are nonzero and of the 
same sign — which for the considered parameter region is the case-one can in principle completely eliminate $\rho_{a b}^{x x}-\rho_{a b}^{y y}$. This is achieved by rescaling the ratio $l_{x} / l_{y}$ by $\sqrt{\rho_{a b}^{y y} / \rho_{a b}^{x x}}$ while at the same time keeping all other model parameters fixed, which leaves the $l_{x}, l_{y}$-independent $\rho_{\perp}$ unchanged [21]. In this way we can realize the model effectively described by Eq. (3). We find that for the lattice geometry illustrated in Fig. 1(a), this rescaling leads to $l_{x}<l_{y}$ in the region of interest, which is consistent with keeping the nearest-neighbor interactions in the $x$ direction only-typical interatomic interactions rapidly decay with increased separation distance. Nevertheless, omitting the nearest-neighbor interactions along the $y$ direction is merely a simplification to reduce the number of system parameters and does not change the main result. Namely, we would like to stress that the presence of vector drag is not limited to systems having nearest-neighbor interaction in one direction only. In what follows we will restrict ourselves to $m=1, n=1 / 2, \quad U=1, U^{\prime}=0.9$, and $V^{\prime}=0.9 V$.

Our findings indicate that in the weakly interacting regime, the macroscopic system harbors a substantial vector drag when $V \neq 0$, at least within our approach. In Fig. 2 we present the analytically derived drag coefficients $\rho_{\perp}$ and $\rho_{\|}$versus $t / U$ and $t^{\prime} / U$, for different values of $V$, in (a), (d), and (g) and in (b), (e), and (h), respectively. The latter quantity is calculated for $l_{x} / l_{y}$ adjusted such that $\rho_{a b}^{x x}=\rho_{a b}^{y y}$, which entails $l_{x}<l_{y}$ apart from the region where $t^{\prime} \gg t$-see panels (c), (f), and (i) of Fig. 2. Since both the magnitude and sign of $\rho_{\perp}$ are determined by the magnitude and sign of $V$, the nearest-neighbor interactions are important for the vector-drag phenomenon in the considered regime.

After demonstrating the effect analytically in the weakcoupling regime, we proceeded to study the model, Eqs. (5) and (6), deep inside the strongly correlated regime. For this purpose, we performed large-scale worm-algorithm Monte Carlo simulations [30,31]. Using a generalization of the Pollock-Ceperley formula [21], which in its original form allows computing the superfluid density through winding number statistics [32], we obtained the dragrelated elements of the more general tensor:

$$
\rho_{a b}^{i j}=\frac{1}{\beta} \frac{l_{i} l_{j}}{l_{x} l_{y}}\left\langle w_{a}^{i} w_{b}^{j}\right\rangle
$$

Here $\beta$ is the inverse temperature, and $\left\langle w_{a}^{i} w_{b}^{j}\right\rangle$ are winding number correlations-the winding number $w_{\alpha}^{i}$ is the net number of times $\alpha$-type particles cross the periodic boundary along the $i$ th direction. Similarly as in the weakly interacting case, we consider the system with constant particle densities equal for both components, i.e., $n_{a}=n_{b}=n=1 / 2$.

The results obtained for the parameters $N=10, t=t^{\prime}$, $\beta=N / t$, and $V / U=0,0.1,0.2$ are presented in Fig. 3 .
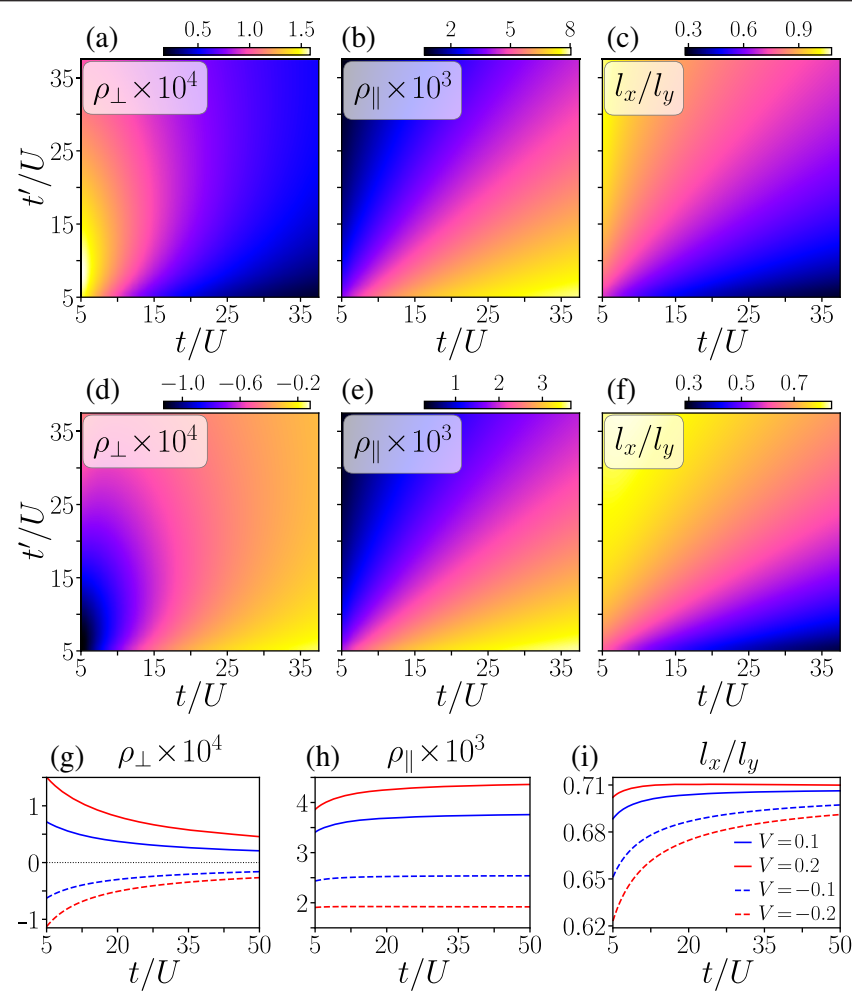

FIG. 2. Vector drag in the weakly interacting regime. The left column (a),(d),(g) presents the $l_{x}, l_{y}$-independent vector-drag coefficient $\rho_{\perp}$. The middle column (b),(e),(h) shows the $l_{x} / l_{y^{-}}$ dependent collinear-drag coefficient $\rho_{\|}$, computed using the ratio $l_{x} / l_{y}$ plotted in the right column (c),(f),(i), which is adjusted such that $\rho_{a b}^{x x}=\rho_{a b}^{y y}$, required for the system to be described by Eq. (3). The upper (a)-(c) and middle (d)-(f) row display how the vector $\operatorname{drag} \rho_{\perp}$, Andreev-Bashkin drag $\rho_{\|}$, and $l_{x} / l_{y}$ depend on $t / U$ and $t^{\prime} / U$ when $V=0.2$ and $V=-0.2$, respectively. Even though the presence of the nearest-neighbor interactions modifies the amplitude of $\rho_{\|}$, it does not change its character determined by the sign. On the contrary, both the sign and magnitude of the vector drag strongly depend on the sign and magnitude of $V$. Note that the sign change of $V$ affects the ratio $\rho_{a b}^{y y} / \rho_{a b}^{x x}$ implying differences between $l_{x} / l_{y}$ visible in (c) and (f). The same quantities, $\rho_{\perp}, \rho_{\|}$and $l_{x} / l_{y}$, but for $t / U=t^{\prime} / U$ and $V= \pm 0.1, \pm 0.2$, are presented in the bottom row (g)-(i).

Here $\rho_{\perp}, \rho_{\|}$, and $\left(\rho_{a b}^{x x}-\rho_{a b}^{y y}\right) / 2$ are plotted against $t / U$ in panels (a)-(c), respectively. At small values of $t / U$, the system is in an insulating phase, which is evident from the coefficients being identically zero. Then, at $t / U \approx 0.053$, the system undergoes a transition into a superfluid state with negative drag coefficients. By further increasing $t / U$, $\rho_{\perp}$ and $\rho_{\|}$increase in value and eventually change sign, whereas $\left(\rho_{a b}^{x x}-\rho_{a b}^{y y}\right) / 2$ reveals a more complex dependence on $t / U$. The behavior of the Andreev-Bashkin drag $\rho_{\|}$is very similar to that of the conventional two-dimensional two-component Bose-Hubbard model [16]. Note that the magnitudes of both $\rho_{\|}$and $\rho_{\perp}$ are maximal in the strongly correlated regime very close to the phase transition point, 

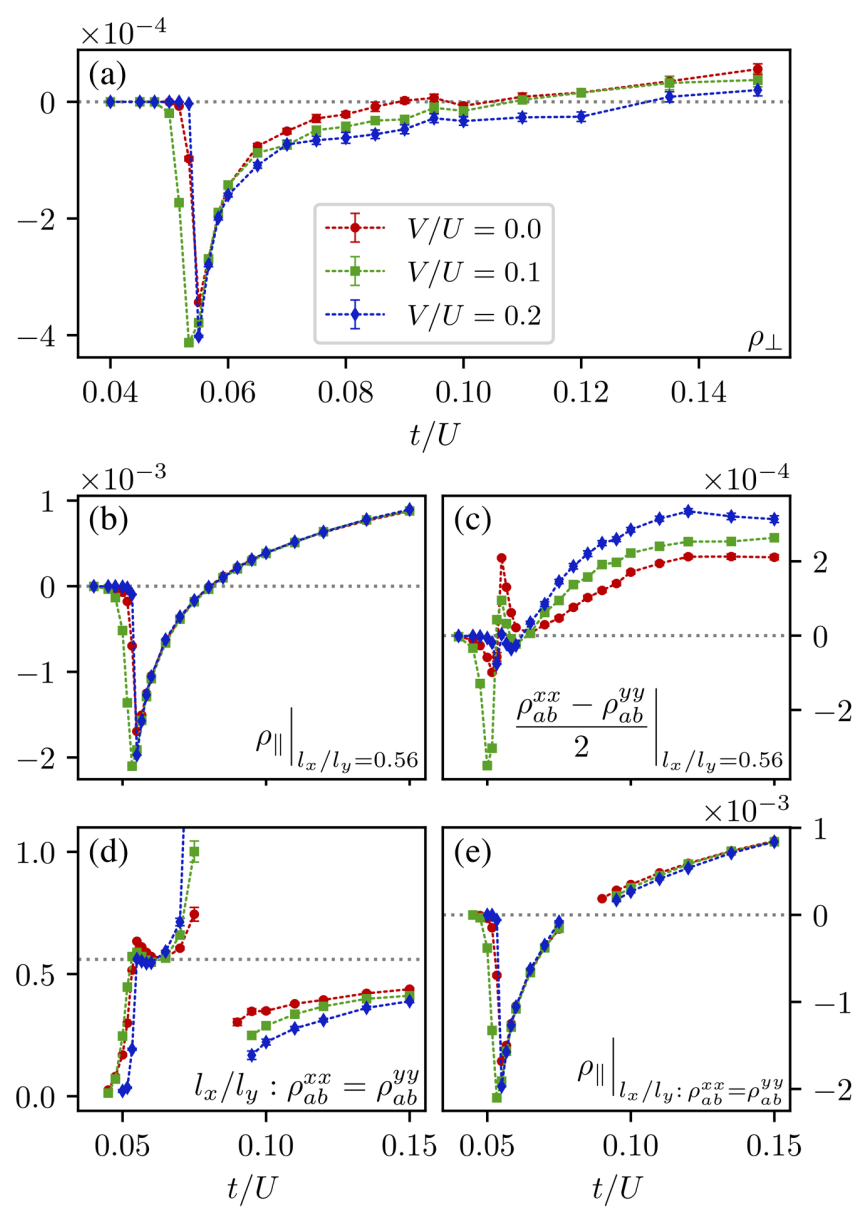

FIG. 3. Vector drag in the strongly interacting regime. The $l_{x}$, $l_{y}$-independent vector-drag coefficient $\rho_{\perp}$ is presented in panel (a), while the $l_{x} / l_{y}$-dependent quantities $\rho_{\|}$and $\left(\rho_{a b}^{x x}-\rho_{a b}^{y y}\right) / 2$ are shown in panels (b) and (c), here using $l_{x} / l_{y}=0.56$. An alternative ratio $l_{x} / l_{y}$-for which $\left(\rho_{a b}^{x x}-\rho_{a b}^{y y}\right) / 2$ is completely eliminated - and the corresponding $\rho_{\|}$are plotted in panels (d) and (e), respectively. In the latter panels, the missing data points around $t / U \approx 0.08$ are due to $\rho_{a b}^{x x}$ and $\rho_{a b}^{y y}$ having opposite signs. All results were obtained using $V / U=0,0.1,0.2 ; t / U=t^{\prime} / U$; $N=10 ;$ and $\beta=N / t$.

which is similar to the fact that in the previously studied systems the Andreev-Bashkin drag is also maximal close to insulating phases [16].

For $l_{x} / l_{y}=0.56$, the difference $\left(\rho_{a b}^{x x}-\rho_{a b}^{y y}\right) / 2$ is comparable to $\rho_{\perp}$ in magnitude and should not be ignored. Nevertheless, by adjusting the $l_{x} / l_{y}$ ratio as previously mentioned we can prompt $\rho_{a b}^{x x}=\rho_{a b}^{y y}$ in situations where $0<\rho_{a b}^{y y} / \rho_{a b}^{x x}<\infty$. This $l_{x} / l_{y}$ ratio, as a function of $t / U$, is shown in panel (d) of Fig. 3, and the resulting $\rho_{\|}$can be found in panel (e). In the vicinity of $t / U \approx 0.08$ the ratio $\rho_{a b}^{y y} / \rho_{a b}^{x x}$ does not fulfill the above-mentioned criteria which is why there are missing data points.

The analytical result for the weakly interacting macroscopic limit, and the unbiased numerical data for the strongly correlated regime, both demonstrate the new type of dissipationless transport manifested in the nonzero value of the vector-drag coefficient $\rho_{\perp}$. In the strong coupling regime, substantial nonzero vector $\operatorname{drag} \rho_{\perp}$ exists in case of exclusively on-site interactions, i.e., for $V=0$, for the system size considered. The vector-drag coefficient's dependence on the system size with purely on-site interactions is further discussed in the Supplemental Material [21], where it is demonstrated that the effect is still present also in larger systems.

In summary, we demonstrated a new dissipationless transport phenomenon in superfluid mixtures in optical lattices. Namely, we have shown that for a class of optical lattices, the free-energy density of an interacting superfluid mixture should contain a vector productlike interaction between the superfluid velocities. This implies that in such a mixture the superflow of each component is not collinear with either of the components' superfluid velocities, i.e., a superflow of one of the components induces a superflow of the other component in the orthogonal direction, in addition to the standard drag. It may be viewed as an intercomponent-interaction-induced superfluid counterpart of the Spin Hall effect.

The strength of the effect was investigated both analytically in the weakly interacting macroscopic limit and numerically in the strongly correlated regime using large-scale worm-algorithm quantum Monte-Carlo simulations. One way to realize this model is through a bilayer optical lattice loaded with dipolar bosons $[33,34]$.

The effect should also be present in multicomponent superconductors. There, at the level of the GinzburgLandau model, it should manifest through the presence of terms in the form of a vectorlike product of components of supercurrents, i.e., mixed terms fourth order in fields and second order in gradients. Investigation of these aspects will be presented in future studies.

E. Bl. and E. Ba. were supported by the Swedish Research Council Grants No. 2016-06122 and No. 201803659, and Göran Gustafsson Foundation for Research in Natural Sciences. A. S and E. Ba. acknowledge the support from Olle Engkvists stiftelse. The computations were enabled by resources provided by the Swedish National Infrastructure for Computing (SNIC) at the National Supercomputer Centre (NSC) partially funded by the Swedish Research Council through Grant No. 2018-05973.

[1] A. Andreev and E. Bashkin, Zh. Eksp. Teor. Fiz. 69, 319 (1975), http://jetp.ras.ru/cgi-bin/e/index/e/42/1/p164?a=list.

[2] A. J. Leggett, Rev. Mod. Phys. 47, 331 (1975).

[3] O. Sjöberg, Nucl. Phys. A265, 511 (1976).

[4] N. Chamel, Mon. Not. R. Astron. Soc. 388, 737 (2008).

[5] M. A. Alpar, S. A. Langer, and J. A. Sauls, Astrophys. J. 282, 533 (1984).

[6] M. G. Alford and G. Good, Phys. Rev. B 78, 024510 (2008).

[7] E. Babaev, Phys. Rev. Lett. 103, 231101 (2009). 
[8] A. B. Kuklov and B. V. Svistunov, Phys. Rev. Lett. 90, 100401 (2003).

[9] A. Kuklov, N. Prokof'ev, and B. Svistunov, Phys. Rev. Lett. 92, 030403 (2004).

[10] A. Kuklov, N. Prokof'ev, and B. Svistunov, Phys. Rev. Lett. 92, 050402 (2004).

[11] A. Kuklov, N. Prokof'ev, B. Svistunov, and M. Troyer, Ann. Phys. (Amsterdam) 321, 1602 (2006), july 2006 Special Issue.

[12] E. V. Herland, E. Babaev, and A. Sudbø, Phys. Rev. B 82, 134511 (2010).

[13] E. K. Dahl, E. Babaev, S. Kragset, and A. Sudbø, Phys. Rev. B 77, 144519 (2008).

[14] M. Greiner, O. Mandel, T. Esslinger, T. W. Hänsch, and I. Bloch, Nature (London) 415, 39 (2002).

[15] I. Bloch, Nat. Phys. 1, 23 (2005).

[16] K. Sellin and E. Babaev, Phys. Rev. B 97, 094517 (2018).

[17] Ş. G. Söyler, B. Capogrosso-Sansone, N. Prokof'ev, and B. Svistunov, New J. Phys. 11, 073036 (2009).

[18] B.Svistunov, E. Babaev, and N. V.Prokofev, Superfluid States of Matter (CRC Press, Boca Raton, FL, 2015), pp. 1-546.

[19] E. K. Dahl, E. Babaev, and A. Sudbø, Phys. Rev. B 78, 144510 (2008).

[20] E. K. Dahl, E. Babaev, and A. Sudbø, Phys. Rev. Lett. 101, 255301 (2008).

[21] See Supplemental Material at http://link.aps.org/ supplemental/10.1103/PhysRevLett.127.100403 for details on the Hamiltonian diagonalization, free energy expansion, generalization of the Pollock-Ceperley formula, Worm-algorithm Monte Carlo method, symmetry analysis, and finite size scaling of the effect in strongly interacting regime.

[22] M. I. D'Yakonov and V. Perel, Sov. J. Exp. Theor. Phys. Lett. 13, 467 (1971), http://jetpletters.ru/ps/1587/ article_24366.shtml.

[23] F. S. Bergeret and I. V. Tokatly, Phys. Rev. B 94, 180502(R) (2016).

[24] J. Linder, M. Amundsen, and V. Risinggård, Phys. Rev. B 96, 094512 (2017).

[25] D. V. Fil and S. I. Shevchenko, Phys. Rev. A 72, 013616 (2005).

[26] J. Linder and A. Sudbø, Phys. Rev. A 79, 063610 (2009).

[27] P. P. Hofer, C. Bruder, and V. M. Stojanović, Phys. Rev. A 86, 033627 (2012).

[28] S. Hartman, E. Erlandsen, and A. Sudbø, Phys. Rev. B 98, 024512 (2018).

[29] J. Nespolo, G. E. Astrakharchik, and A. Recati, New J. Phys. 19, 125005 (2017).

[30] N. V. Prokof'ev, B. V. Svistunov, and I. S. Tupitsyn, J. Exp. Theor. Phys. 87, 310 (1998).

[31] B. Capogrosso-Sansone, N. V. Prokof'ev, and B. V. Svistunov, Phys. Rev. B 75, 134302 (2007).

[32] E. L. Pollock and D. M. Ceperley, Phys. Rev. B 36, 8343 (1987).

[33] A. Macia, G. E. Astrakharchik, F. Mazzanti, S. Giorgini, and J. Boronat, Phys. Rev. A 90, 043623 (2014).

[34] A. Safavi-Naini, Ş. G. Söyler, G. Pupillo, H. R. Sadeghpour, and B. Capogrosso-Sansone, New J. Phys. 15, 013036 (2013). 